\title{
Impact of Ciprofloxacin and Chloramphenicol on the Lipid Bilayer of Staphylococcus aureus: Changes in Membrane Potential
}

\author{
Paulina L. Páez, María C. Becerra, and Inés Albesa \\ Departamento de Farmacia, Facultad de Ciencias Químicas, Universidad Nacional de Córdoba, Haya de la Torre y Medina Allende, \\ Ciudad Universitaria, IMBIV-CONICET, 5000 Córdoba, Argentina \\ Correspondence should be addressed to Paulina L. Páez; plpaez@fcq.unc.edu.ar
}

Received 18 March 2013; Revised 16 April 2013; Accepted 7 May 2013

Academic Editor: Afaf K. El-Ansary

Copyright (C) 2013 Paulina L. Páez et al. This is an open access article distributed under the Creative Commons Attribution License, which permits unrestricted use, distribution, and reproduction in any medium, provided the original work is properly cited.

\begin{abstract}
The present study was undertaken to explore the interaction of ciprofloxacin and chloramphenicol with bacterial membranes in a sensitive and in a resistant strains of Staphylococcus aureus by using 1-anilino-8-naphthalene sulfonate (ANS). The binding of this probe to the cell membrane depends on the surface potential, which modulates the binding constant to the membrane. We observed that these antibiotics interacted with the bilayer, thus affecting the electrostatic surface potential. Alterations caused by antibiotics on the surface of the bacteria were accompanied by a reduction in the number of binding sites and an increase in the ANS dissociation constant in the sensitive strain, whereas in the ciprofloxacin-resistant strain no significant changes were detected. The changes seen in the electrostatic surface potential generated in the membrane of $S$. aureus by the antibiotics provide new aspects concerning their action on the bacterial cell.
\end{abstract}

\section{Introduction}

The plasmatic membrane is a chemoosmotic barrier that provides an interface between the organism and the environment. This bilayer presents an electrochemical potential (negative in the interior) which plays a basic role in the control of the exchange of solutes. Disturbances in the membrane potential can provide a rapid and sensitive indication of those stimuli that lead to physiological functionally important changes with respect to bacterial viability [1].

Fluorescent molecules have been extensively used as probes of biological membranes. These hydrophobic and amphiphilic probes are associated with membranes when added to cells or artificial systems, and their resultant fluorescence properties can be used to monitor a variety of membrane characteristics. In general, the addition of effectors results in the deenergization of cells, which leads to increased fluorescence from the probes present in the cell suspension, such as negatively charged 8-anilino-1-naphthalenesulfonate (ANS) [2].

ANS binding and fluorescence strongly respond to modulation of the surface potential, with the energy-dependent quenching being largely due to the generation of $\Delta \Psi$ and being accounted for by the movement of the anion across the membrane and from intramembrane sites in response to membrane potential [3].

It has been demonstrated that the determination of the membrane potential based on fluorochromes provides a useful and sensitive approximation for the monitoring of the cellular stresses in bacteria [4-6], since both oxidative and nitrosative stress are able to depolarize the plasmatic membrane [7].

The effect of the oxidative stress generated by reactive oxygen species (ROS) has been described as one of the most important sources of metabolic disturbance and the cellular damage. These agents are involved in the first important changes in the plasmatic membrane, and consequently at the beginning of cellular death [8-10].

Bacterial gyrase inhibitors, including synthetic quinolone antibiotics, induce a breakdown in iron regulatory dynamics, which promotes the formation of the ROS that contribute to cell death [11].

Bactericidal antibiotic killing mechanisms are currently attributed to the class of specific drug-target interactions. 
However, the understanding of many of the bacterial responses that occur as a consequence of the primary drugtarget interaction remains incomplete. It is known that oxidative stress in bacteria can be caused by exogenous agents that originate toxic effects, and our previous studies have shown that ciprofloxacin (CIP) and chloramphenicol (CMP), among others, can stimulate the induction of ROS in different bacterial species [12-16].

The aim of the present study was to explore the effects of clinically used antibiotics such as CIP and CMP on the lipid surface and to estimate the variation in the membrane potential in Staphylococcus aureus strains.

\section{Materials and Methods}

2.1. Susceptibility Determination. The antimicrobial activities of CIP and CMP were evaluated in two strains, one standard strain S. aureus ATCC 29213 and other clinical strain S. aureus by using the standard tube dilution method following the indications of the Clinical and Laboratory Standards Institute [17]. The strains were maintained by culture in trypticase soy broth (TSB) for $24 \mathrm{~h}$ at $37^{\circ} \mathrm{C}$, and the minimum inhibitory concentration (MIC) was determined by using the standard tube dilution method. Cultures of $24 \mathrm{~h}$ in Mueller-Hinton medium were diluted to $10^{6} \mathrm{CFU} / \mathrm{mL}$, incubated for $10 \mathrm{~min}$ at $37^{\circ} \mathrm{C}$, and then the antibiotics were added at different concentrations $(0.125 \mu \mathrm{g} / \mathrm{mL}-512 \mu \mathrm{g} / \mathrm{mL})$. Bacterial growth was observed at $24 \mathrm{~h}$ of incubation. MIC was determined as the lowest antibiotic concentrations at which growth was completely inhibited after overnight incubation of the tubes at $37^{\circ} \mathrm{C}$. MICs were determined three times and the median values are taken.

2.2. ANS Binding Studies. Overnight cultures of S. aureus ATCC 29213 and clinical strain S. aureus were prepared in trypticase soy broth. Suspensions were centrifuged, and the pellets were resuspended in saline phosphate buffer (PBS) $\mathrm{pH}$ 7.4 at an optical density of 0.4 at $600 \mathrm{~nm}$. Then, $50 \mu \mathrm{L}$ of these suspensions was incubated with $256 \mu \mathrm{g} / \mathrm{mL}$ of CIP, $4 \mu \mathrm{g} / \mathrm{mL}$ of CMP, or without antibiotic (control) in a total volume of $1 \mathrm{~mL}$ in PBS.

The suspensions were centrifuged, and $1 \mathrm{~mL}$ of Triton $1 \%$ $\mathrm{V} / \mathrm{V}$ in $\mathrm{NaCl} 10 \%$ was added to the pellet. Then, $20 \mu \mathrm{L}$ of ANS $60 \mu \mathrm{M}$ was added to $50 \mu \mathrm{L}$ of bacterial suspensions and PBS to a total volume of $3 \mathrm{~mL}$. The assay mixture for the standard curve consisted of $1 \mathrm{~mL}$ of bacterial suspensions and different concentrations of ANS, ranging from 0 to $120 \mu \mathrm{M}$. The structural changes on the membrane potential were studied by using L-anilino naphthalene-8-sulphonate as the fluorescent probe by the method of Verma et al. [18] and Robertson and Rottenberg [19]. The fluorescence emission was recorded on a Spectrofluorometer PTI (Photon Technology International) Model Quanta Master 2 QM2, with phosphorescence lifetime measurements taken at excitation and emission wavelengths of $360 \mathrm{~nm}$ and $516 \mathrm{~nm}$, respectively. These experiments were performed at room temperature $\left(23^{\circ} \mathrm{C}\right)$.

2.3. Measurement of $K_{d}$ and $n$ from ANS by Fluorescence Emission in Bacteria. The approach used to determine the dissociation constant $\left(K_{d}\right)$ and the number of binding sites (n) from the fluorescence yield was as previously reported by Verma et al. [18] and Robertson and Rottenberg [19]. The fluorescence developed is recorded, and the data is plotted as the reciprocal of the fluorescence signal (arbitrary units) versus the reciprocal of the concentration of ANS. This produces a straight line whose extrapolation with the ordinate gives the reciprocal of the limiting fluorescence of ANS $\left(F_{\max }\right)$. The number of binding sites for ANS was calculated by plotting the bound ANS per mg protein/free ANS versus bound ANS per mg protein.

2.4. Statistical Analysis. The assays were carried out at least in triplicate. Data were expressed as mean \pm SD and analyzed by the Student's $t$-test. $P<0.05$ was used as the level of statistical significance.

\section{Results and Discussion}

S. aureus ATCC 29213 exhibited sensitivity to CIP and CMP, with MICs of $0.5 \mu \mathrm{g} / \mathrm{mL}$ for CIP and $1 \mu \mathrm{g} / \mathrm{mL}$ for CMP. In addition, the clinical strain $S$. aureus MICs obtained were $32 \mu \mathrm{g} / \mathrm{mL}$ for CIP and $8 \mu \mathrm{g} / \mathrm{mL}$ for CMP; according to these results, the strain was resistant to CIP but sensitive to CMP. The fluorescence emission of ANS at $516 \mathrm{~nm}$ in the presence of $256 \mu \mathrm{g} / \mathrm{mL}$ of CIP, $4 \mu \mathrm{g} / \mathrm{mL}$ of CMP or in the absence of antibiotic was determined with S. aureus ATCC 29213 and clinical strain $S$. aureus.

Data were plotted as the reciprocal of the fluorescence signal $(1 / \mathrm{F})$ versus the reciprocal of the concentration of free ANS (1/ANS), as the reciprocal of the intercept gives the limit of the ANS fluorescence $\left(F_{\max }\right)$, a parameter related to the maximum concentration of bound ANS. From the slope, the $K_{d}$ was obtained from which the affinity of the fluorescent probe for binding sites on the bilayer could be inferred.

The values of bound ANS and free ANS were calculated from (1), where

$$
\begin{gathered}
\text { ANS bound }(\mathrm{nmol})=\frac{1 / F_{\max }}{1 / F} \times 100, \\
\mathrm{ANS}_{\text {free }}(\mathrm{nmol})=\mathrm{ANS}_{\text {total }}-\mathrm{ANS}_{\text {bound }} .
\end{gathered}
$$

The surface potential $(\Psi)$ was calculated according to (2):

$$
\Psi=59 \log \left(F / F_{o}\right)
$$

where $F$ corresponds to the fluorescence in the presence of each antibiotic and $F_{o}$ corresponds to the fluorescence obtained when $\Psi=0$.

The change in membrane potential $(\Delta \Psi)$ in the presence of antibiotic with respect to the control without antibiotic was obtained from the difference between $\Psi$ with and without antibiotic (3):

$$
\Delta \Psi=\Psi-\Psi_{\text {control }}
$$

The number of binding sites for ANS was calculated by using (4), in which the intercept represents the value of the 
TABLE 1: Parameters obtained from the ANS binding studies in S. aureus ATCC 29213 and clinical strain S. aureus.

\begin{tabular}{|c|c|c|c|c|}
\hline$F_{\max }$ & $K_{d}$ & $n$ & $\Psi(\mathrm{mV})$ & $\Delta \Psi(\mathrm{mV})$ \\
\hline \multicolumn{5}{|c|}{ S. aureus ATCC 29213 sensitive to CIP and CMP } \\
\hline Control without antibiotic 143 & 251 & 603028 & -306 & - \\
\hline Ciprofloxacin $256 \mu \mathrm{g} / \mathrm{mL}$ & 927 & 52983 & -406 & -100 \\
\hline Chloramphenicol $4 \mu \mathrm{g} / \mathrm{mL} 62$ & 846 & 48332 & -331 & -25 \\
\hline \multicolumn{5}{|c|}{ Clinical strain S. aureus resistant to CIP } \\
\hline Control without antibiotic 77 & 902 & 45110 & -287 & - \\
\hline Ciprofloxacin $256 \mu \mathrm{g} / \mathrm{mL} \quad 77$ & 937 & 46865 & -302 & -15 \\
\hline \multicolumn{5}{|c|}{$\begin{array}{l}F_{\max } \text { is the fluorescence intensity (related to the maximum concentration of } \\
\text { bound ANS), } n \text { is the number of binding sites of ANS to the membrane, } K_{d} \text { is } \\
\text { the dissociation constant, } \Psi \text { is the potential at the surface of the membrane } \\
\text { and } \Delta \Psi \text { is the change in surface potential of the membrane in the control } \\
\text { without antibiotic. }\end{array}$} \\
\hline
\end{tabular}

reciprocal of $q$ (a constant of proportionality), with the value of $n$ being obtained from the slope $\left(K_{d} / q n\right)$ :

$$
\frac{\mathrm{ANS}_{\text {free }}}{F}=\frac{K_{d}}{q^{n}} \times \frac{1}{\text { [bacteria] }}+\frac{1}{q} .
$$

The same procedure was performed to determine the number of binding sites for ANS when the bacterial suspensions were incubated with the antibiotics studied, where a decrease in the number of binding sites for ANS with antibiotic would suggest changes in the cell surface.

Any alteration in the binding of ANS to the membrane of $S$. aureus in the presence of antibiotics was tested by a decrease in the number of binding sites for ANS compared with control. When ANS bounds to phospholipids, the fluorescence intensity increased with high concentrations of ANS. However, in the presence of CMP or CIP, the ANS fluorescence decreased, with this behavior reflecting competition between CMP and the ANS binding site that might have been located at the interface of the membrane. By comparing the values of $K_{d}$, we were able to infer that the affinity of the probe sites of the bilayer was affected by the two antibiotics. Moreover, differences in the values of the electric potentials indicated alterations in the bacterial membranes.

The fluorescence of ANS emission was determined at $516 \mathrm{~nm}$ in the presence of CIP and CMP and in the absence of antibiotic. The data were plotted as the reciprocal of the fluorescence signal $(1 / F)$ versus the reciprocal of the concentration of ANS (1/ANS) (Figure 1). There was a linear relationship between fluorescence and the inverse of the concentration of bound ANS, revealing a change in the affinity of the membrane in the presence of antibiotic with respect to control.

Table 1 shows the parameters obtained with CMP and CIP for the surface potential in the absence or presence of the antibiotics in the S. aureus ATCC 29213 and clinical strain S. aureus.

Whereas the value of $F_{\max }$ was reduced twofold and the number of binding sites decreased elevenfold, the $K_{d}$ value was increased fourfold, compared to the control without antibiotic in S. aureus ATCC 29213 (sensitive to CIP). In clinical strain $S$. aureus (resistant to CIP) there were no

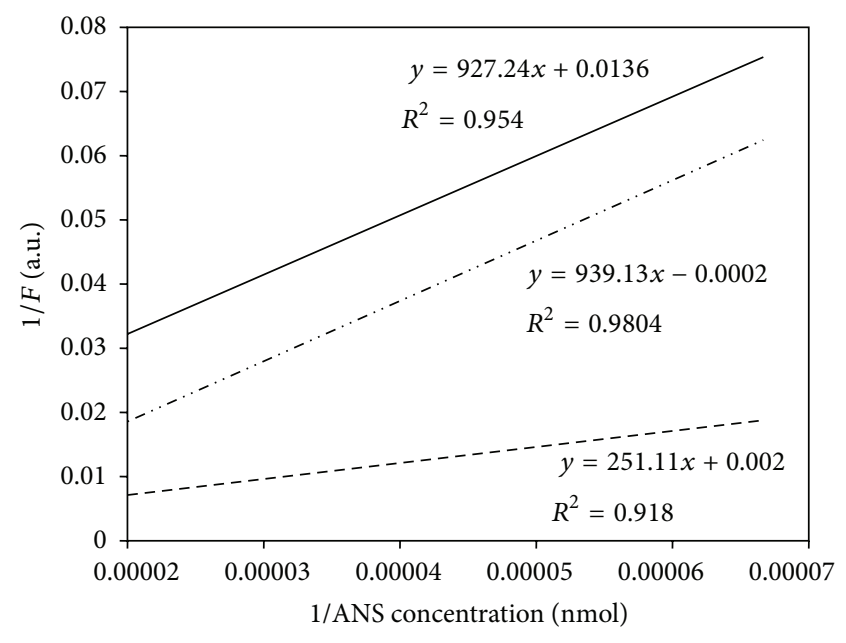

FIgURE 1: Scatchard plots of ANS interaction with S. aureus ATCC 29213 control (- - ), treated with ciprofloxacin (-) and treated with CMP (-.-).

significant changes in $F_{\max }$, number of binding sites, or the $K_{d}$ value. The baseline value of $\Psi$ in the sensitive strain was $-306 \mathrm{mV}$, while in the resistant strain this was $-287 \mathrm{mV}$. The $\Delta \Psi$ was almost seventimes higher in the sensitive strain $(100 \mathrm{mV})$ than in the resistant one $(15 \mathrm{mV})$. CIP, a bactericidal antibiotic, reduced this value by $33 \%$ in the sensitive strain, while the reduction in the resistant one was only $5 \%$. Finally, CMP, a bacteriostatic antibiotic, increased the membrane potential by about $11 \%$ in the sensitive strain.

The membrane potential is an important parameter that controls various cellular processes. It is a sensitive indicator of energy status and cell viability, with membrane depolarization leading to excessive production of ROS which is an indication of an advance in cellular dysfunction and precedes many other signs of cellular injury. A reduction in the potential also provides information about the feasibility of transferring an electron "in vivo." In addition, the catalytic production of oxidative stress from the redox cycle is a possible mode of action of antibiotics, because it could indicate interference with the electron transport chain $[20$, 21].

The changes in the electric potential obtained in the present work showed alterations in the bacterial membrane of $S$. aureus in the presence of CMP and CIP. Furthermore, changes caused by antibiotics on the surface of the bacteria were demonstrated by a reduction in the number of binding sites of the fluorescent probe and an increase in the ANS dissociation constant.

Montero et al. established that CIP interacts with neutral and charged membranes at the surface level (headgroup region). They also postulated that this could be part of the mechanism of entry of the 6-fluoroquinolones through the cytoplasmic membrane [3]. Ciprofloxacin is a widely used antimicrobial agent against Gram positive and Gram negative, but there are conflicting reports about the effect of CIP on the bacterial membrane $[22,23]$.

There are previous results on S. aureus, with liposomes of E. coli showing interaction of ANS with a lipid bilayer and in 
the presence of 6-FQs as a result of a reduction in the maximum concentration of the ANS bound to liposomes [2427]. However, there are no comparisons reported between sensitive and resistant strains in the presence of antibiotics.

The present results demonstrated that the strains had a particular behavior in the presence of each antibiotic, an effect that was manifested by the differences obtained in the bacterial membrane potential. Moreover, on comparing the sensitive strain with the resistant one, a higher alteration in the membrane potential was observed in sensitive bacterium that was associated with the effect of the antibiotic [20,21].

\section{Conclusions}

In our previous reports, we demonstrated that CIP and CMP induce oxidative stress in $S$. aureus strains, with sensitive clinical strains producing higher ROS levels than resistant ones [12-15]. The present work shows that these antibiotics had an impact on the lipid bilayer, leading to significant membrane potential changes in $S$. aureus sensitive to ciprofloxacin. These observations can be added to the mechanism of action previously described for the antibiotics investigated, with the changes generated in the lipid bilayers of $S$. aureus contributing new aspects about the action of these antibiotics on the bacterial cell.

\section{Authors' Contribution}

Paulina L. Páez and María C. Becerra contribute equally to this work.

\section{Acknowledgments}

The authors thank Dr. Gerardo Argüello (Departamento de Físicoquímica, Facultad de Ciencias Químicas, UNC, INFIQC-CONICET) for assistance with the fluorescence measurements. The authors thank Consejo Nacional de Investigaciones Científicas y Técnicas de Argentina (CONICET), Secretaría de Ciencia y Técnica de la Universidad Nacional de Córdoba (SECyT) for financial support. We thank native English speaker Dr. Paul Hobson for revision of this manuscript. Paulina L. Páez and María C. Becerra are members of the Research Career of CONICET.

\section{References}

[1] F. David, M. Hebeisen, G. Schade, E. Franco-Lara, and M. Di Berardino, "Viability and membrane potential analysis of Bacillus megaterium cells by impedance flow cytometry," Biotechnology \& Bioengineering, vol. 109, no. 2, pp. 483-492, 2012.

[2] M. K. Wolf and J. Konisky, "Increased binding of a hydrophobic, photolabile probe to Escherichia coli inversely correlates to membrane potential but not adenosine 5'-triphosphate levels," Journal of Bacteriology, vol. 145, no. 1, pp. 341-347, 1981.

[3] M. T. Montero, M. Pijoan, S. Merino-Montero, T. Vinuesa, and J. Hernández-Borrell, "Interfacial membrane effects of fluoroquinolones as revealed by a combination of fluorescence binding experiments and atomic force microscopy observations," Langmuir, vol. 22, no. 18, pp. 7574-7578, 2006.

[4] M. G. Dinsdale, D. Lloyd, and B. Jarvis, "Yeast vitality during cider fermentation: two approaches to the measurement of membrane potential," Journal of the Institute of Brewing, vol. 101, no. 6, pp. 453-458, 1995.

[5] D. J. Mason, R. Allman, J. M. Stark, and D. Lloyd, "Rapid estimation of bacterial antibiotic susceptibility with flow cytometry," Journal of Microscopy, vol. 176, no. 1, pp. 8-16, 1994.

[6] D. Lloyd, D. J. Mason, and M. T. E. Suller, "Microbial infections," in Cytometric Analysis of Cell Phenotype and Function, D. A. McCarthy and M. G. Macey, Eds., pp. 2982-3213, Cambridge University Press, Cambridge, UK, 1st edition, 2001.

[7] D. Lloyd, J. C. Harris, G. A. Biagini et al., “The plasma membrane of microaerophilic protitsts: oxidative and nitrosative stress," Microbiology, vol. 150, no. 5, pp. 1183-1190, 2004.

[8] J. A. Scott and C. A. Rabito, "Oxygen radicals and plasma membrane potential," Free Radical Biology and Medicine, vol. 5, no. 4, pp. 237-246, 1988.

[9] M. Kayahara, U. Felderhoff, J. Pocock, M. N. Hughes, and H. Mehmet, "Nitric oxide (NO) and the nitrosonium cation $\left(\mathrm{NO}^{+}\right)$ reduce mitochondrial membrane potential and trigger apoptosis in neuronal PC12 cells," Biochemical Society Transactions, vol. 26, no. 4, p. S340, 1998.

[10] D. Lloyd, J. C. Harris, S. Maroulis et al., "Nitrosative stress induced cytotoxicity in Giardia intestinalis," Journal of Applied Microbiology, vol. 95, no. 3, pp. 576-583, 2003.

[11] M. A. Kohanski, D. J. Dwyer, B. Hayete, C. A. Lawrence, and J. J. Collins, "A common mechanism of cellular death induced by bactericidal antibiotics," Cell, vol. 130, no. 5, pp. 797-810, 2007.

[12] I. Albesa, M. C. Becerra, P. C. Battán, and P. L. Páez, “Oxidative stress involved in the antibacterial action of different antibiotics," Biochemical and Biophysical Research Communications, vol. 317, no. 2, pp. 605-609, 2004.

[13] M. C. Becerra, P. L. Páez, L. E. Laróvere, and I. Albesa, "Lipids and DNA oxidation in Staphylococcus aureus as a consequence of oxidative stress generated by ciprofloxacin," Molecular and Cellular Biochemistry, vol. 285, no. 1-2, pp. 29-34, 2006.

[14] P. L. Páez, M. C. Becerra, and I. Albesa, "Antioxidative mechanisms protect resistant strains of Staphylococcus aureus against ciprofloxacin oxidative damage," Fundamental and Clinical Pharmacology, vol. 24, no. 6, pp. 771-776, 2010.

[15] V. Aiassa, A. I. Barnes, and I. Albesa, "Resistance to ciprofloxacin by enhancement of antioxidant defenses in biofilm and planktonic Proteus mirabilis," Biochemical and Biophysical Research Communications, vol. 393, no. 1, pp. 84-88, 2010.

[16] P. L. P. Páez, M. C. Becerra, and I. Albesa, "Comparison of macromolecular oxidation by reactive oxygen species in three bacterial genera exposed to different antibiotics," Cell Biochemistry and Biophysics, vol. 61, no. 3, pp. 467-472, 2011.

[17] Clinical and Laboratory Standards Institute, Performance standards for antimicrobial susceptibility testing, 18th informational supplement, Clinical and Laboratory Standards Institute), p. approved standard M100-S18, Clinical and Laboratory Standards Institute, Wayne, Pennsylvania, 2008.

[18] I. Verma, A. Rohilla, and G. K. Khuller, "Alterations in macromolecular composition and cell wall integrity by ciprofloxacin in Mycobacterium smegmatis," Letters in Applied Microbiology, vol. 29, no. 2, pp. 113-117, 1999.

[19] D. E. Robertson and H. Rottenberg, "Membrane potential and surface potential in mitochondria. Fluorescence and binding 
of 1-anilinonaphthalene-8-sulfonate," The Journal of Biological Chemistry, vol. 258, no. 18, pp. 11039-11048, 1983.

[20] N. Zamzami, T. Hirsch, B. Dallaporta, P. X. Petit, and G. Kroemer, "Mitochondrial implication in accidental and programmed cell death: apoptosis and necrosis," Journal of Bioenergetics and Biomembranes, vol. 29, no. 2, pp. 185-193, 1997.

[21] A. V. Gyulkhandanyan, C. J. Feeney, and P. S. Pennefather, "Modulation of mitochondrial membrane potential and reactive oxygen species production by copper in astrocytes," Journal of Neurochemistry, vol. 87, no. 2, pp. 448-460, 2003.

[22] A. Dalhoff, "Interaction of aminoglycosides and ciprofloxacin with bacterial membrane," in The Influence of Antibiotics on the Host Parasite Relationship, H. Adam, W. Hahn, and W. Opferkuch, Eds., pp. 16-27, Springer, Berlin, Germany, 1985.

[23] C. S. Lewin, B. M. A. Howard, and J. T. Smith, "Proteinand RNA-synthesis independent bactericidal activity of ciprofloxacin that involves the a subunit of DNA gyrase," Journal of Medical Microbiology, vol. 34, no. 1, pp. 19-22, 1991.

[24] S. Merino, J. L. Vázquez, O. Domènech et al., "Fluoroquinolonebiomembrane interaction at the DPPC/PG lipid-bilayer interface," Langmuir, vol. 18, no. 8, pp. 3288-3292, 2002.

[25] J. L. Vázquez, M. T. Montero, S. Merino et al., "Location and nature of the surface membrane binding site of ciprofloxacin: a fluorescence study," Langmuir, vol. 17, no. 4, pp. 1009-1014, 2001.

[26] A. Grancelli, A. Morros, M. E. Cabañas et al., "Interaction of 6fluoroquinolones with dipalmitoylphosphatidylcholine monolayers and liposomes," Langmuir, vol. 18, pp. 9177-9182, 2002.

[27] O. Domenech, Y. F. Dufrêne, F. Van Bambeke, P. M. Tukens, and M. P. Mingeot-Leclercq, "Interactions of oritavancin, a new semi-synthetic lipoglycopeptide, with lipids extracted from Staphylococcus aureus," Biochimica et Biophysica Acta, vol. 1798, no. 10, pp. 1876-1885, 2010. 

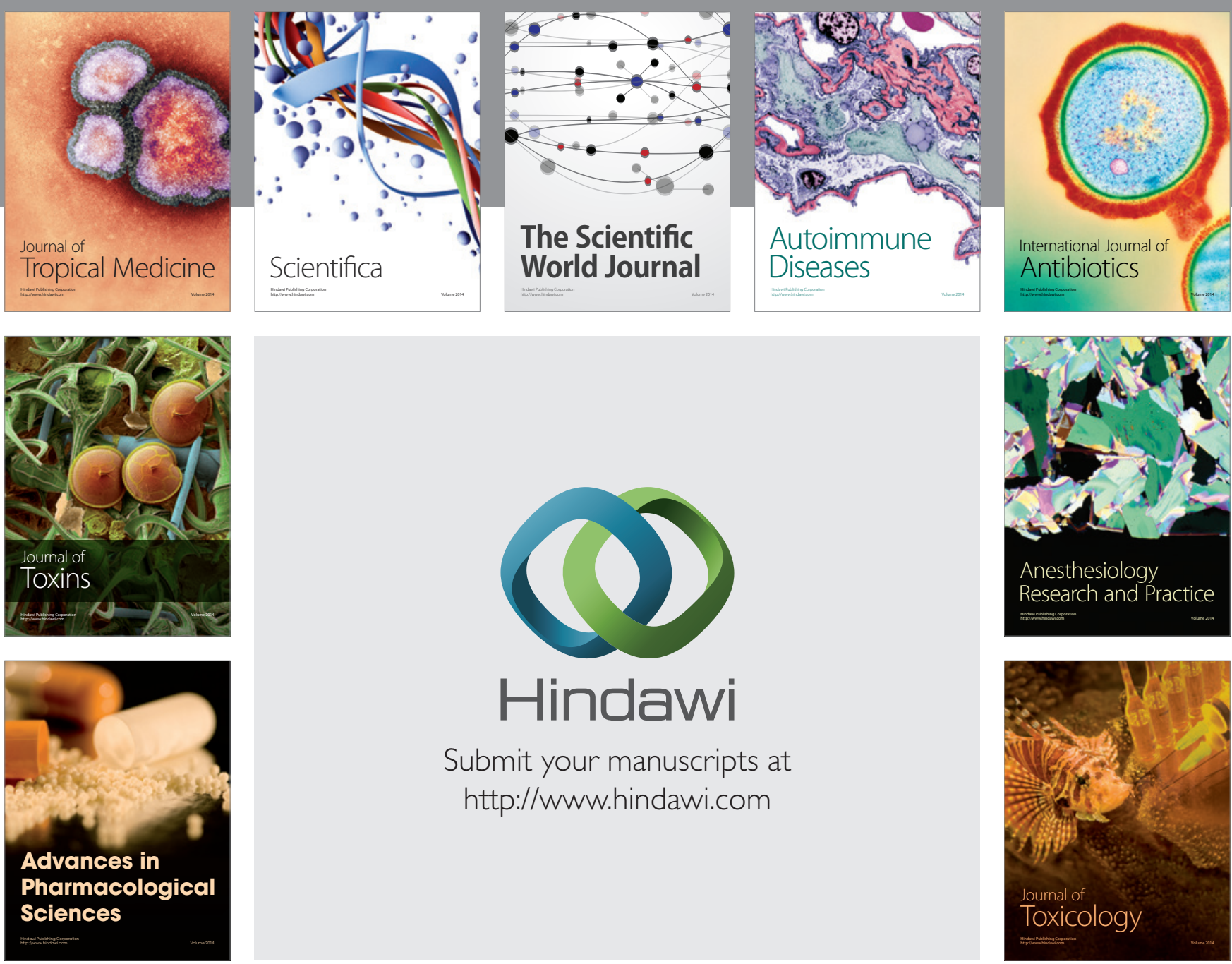

\section{Hindawi}

Submit your manuscripts at

http://www.hindawi.com
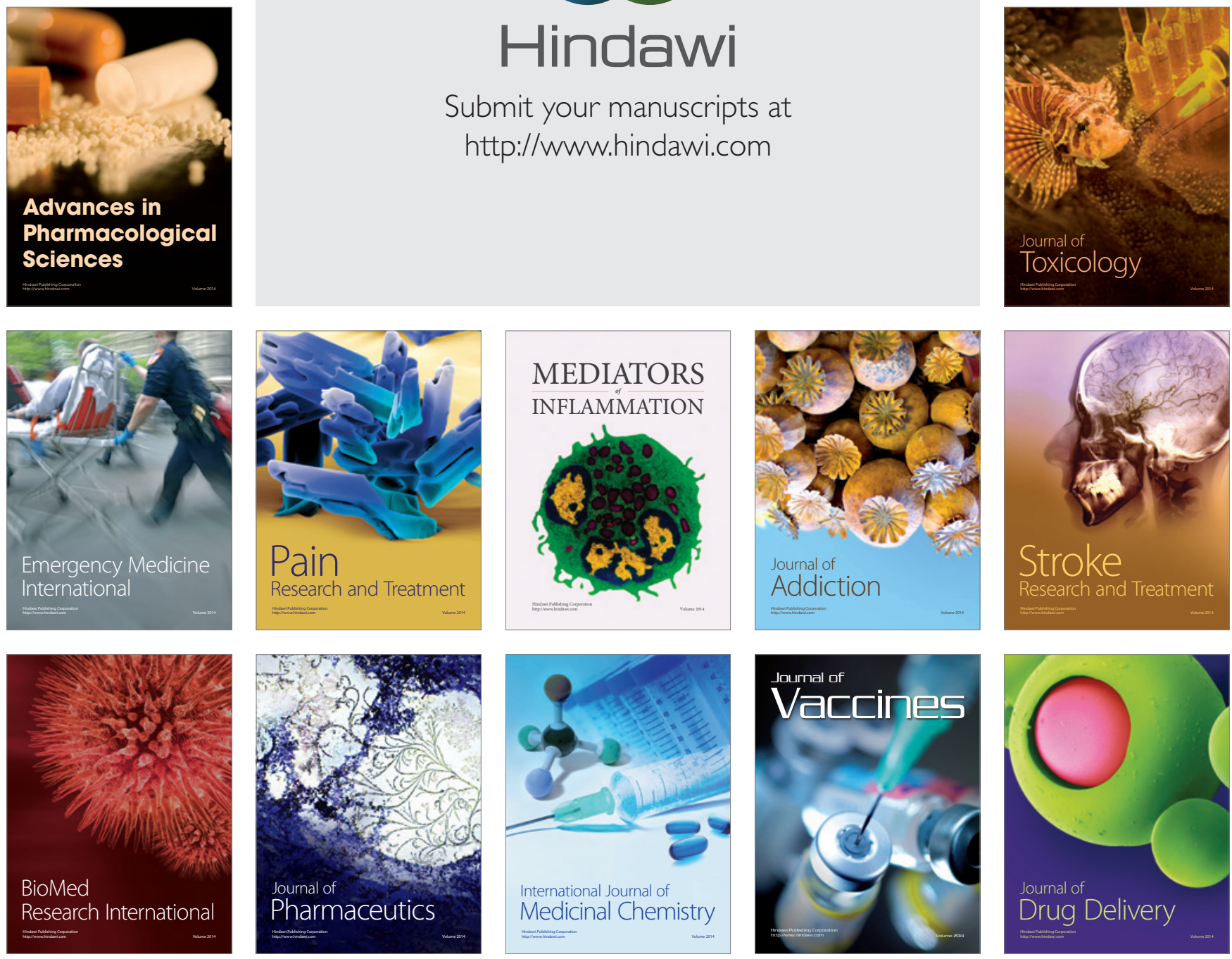